\title{
Human Permanent Denervated Muscles Recovery
}

\author{
Contributors: , Ugo Carraro ${ }^{1}$, ( Paolo Gargiulo ${ }^{2}$, ( Jorgelina Ramos ${ }^{3}$, Romain \\ Aubonnet ${ }^{4}$, Helmut Kern ${ }^{5}$ \\ 1, Department of Biomedical Sciences, University of Padova, Via Ugo Bassi, 58/B 35131 \\ Padova, Italy; ugo.carraro@unipd.it \\ 2, Institute for Biomedical and Neural Engineering, Department of Science, Landspitali \\ University Hospital, University of Iceland; paologar@landspitali.is \\ 3, jorjita.ramos@gmail.com \\ 4, romaina@ru.is \\ 5, Physiko- und Rheumatherapie, Neugebäudeplatz 1, A-3100 St. Pölten, Austria;
} helmut@kern-reha.at

Version received: 2 September 2020

check for updates

\section{Definition}

We demonstrated the long-term clinical value of co-activating thigh muscles through hbFES strategy using high currents and large surface electrodes. This Vienna Strategy is able to reverse, at clinically relevant levels, the adverse effects of Spinal Cord Injury (SCl), even in the worst-case scenario of complete lesion of lower motor neurons, as it may occur in complete conus and cauda equina syndrome. Continued regularly, hbFES for denervated, degenerating muscles helps to maintain healthier leg muscles and skin, reducing the risks of lifethreatening SCl complications.

By products of these studies are new approaches for counteracting aging muscle atrophy, new color clinical imaging of muscle tissue and Machine Learning Predictive Systems for skeletal muscle diagnostics and managements.

\section{Introduction}

Muscle atrophy is a muscle loss that occurs inexorably during late aging ${ }^{[1][2]}$ or earlier with prolonged malnutrition, bed rest, neural and skeletal muscle injuries, chronic cardiovascular and respiratory failures, diabetes, sepsis, and cancer. It is a dismal truth that human muscles naturally decline from the age of 30 years and there are innumerable neuromuscular disorders, which induce muscle atrophy, impairing the mobility of patients. III and elderly people generally spend very little time in daily physical activity. The resulting disuse atrophy limits more and more patient independence confining people to wheelchairs, beds, and to prolonged hospitalizations ${ }^{[3]}$. Together with septic and oncologic cachexia ${ }^{[4]}$, extreme sarcopenia characterizes complete, irreversible denervation of skeletal muscles, whose early stages of mild and severe atrophy evolve finally in fibro-fatty degeneration of muscle tissue ${ }^{[5]}$.

\subsection{Counteracting muscle atrophy}

Severely ill people, and even the extreme elderly, may counteract muscle deterioration by working to maintain the majority of their skeletal muscles in the best possible shape [6]7][8]. All chronic and progressive muscle impairments need permanent management. An effective and low-cost option is to educate people and patients on how to do physical activity, regardless of whether it is volitional [9] or electrical stimulation-induced exercise for those persons, which are not willing or able to move as needed [10][11]. Cardiovascular and respiratory rehabilitation for patients actually aims to reverse muscle atrophy and weakness [12][13]. Balance between costs and benefits is debated ${ }^{[14][15]}$, but many practitioners recognize the value of electrical stimulation in their daily clinical practice $[16][17][18][19]$.

Direct spinal cord stimulation promises to revolutionize the management of thoracic-level spinal cord injury (SCl) patients [20]. Unfortunately, this approach may be applied only to SCI patients with upper motor neuron lesion. While this approach deserves further validation and dissemination, there are $\mathrm{SCl}$ patients with complete lower motor neuron 
lesions (e.g., those suffering complete lesions of conus and cauda), which cannot be enrolled, i.e., those with permanent, irreversible denervation of skeletal muscles.

In the latter peculiar cases, we will show that it is possible to recover functional standing exercises by a surface homebased functional electric stimulation (hbFES) rehabilitation strategy [21]. The high intensity of the hbFES regime required to activate permanently denervated muscles is extremely painful for normal persons, and therefore is limited to patients with loss of peripheral sensation as it occurs in the complete lesion of conus and cauda equina.

Here we add that the purpose-developed electrical fields delivered to ventral aspects of the thigh muscles by anatomically shaped large surface electrodes ${ }^{[22]}$ produce clinically relevant recovery of the hamstring muscles ${ }^{[23]}$ and of the thigh skin [24][25][26]. A believed drawback, co-activation of non-targeted muscles, is indeed responsible for a positive clinical result.

\subsection{Evidence of permanent long-term denervation}

Complete and permanent denervation of right and left quadriceps muscles was assessed before and after two years of hbFES by test electrical stimulation (Figure 1), needle electromyography, and both transcranial and lumbosacral magnetic stimulation, as previously described [Quantitative Muscle Color Computed Tomography in compliant and non-compliant patients].
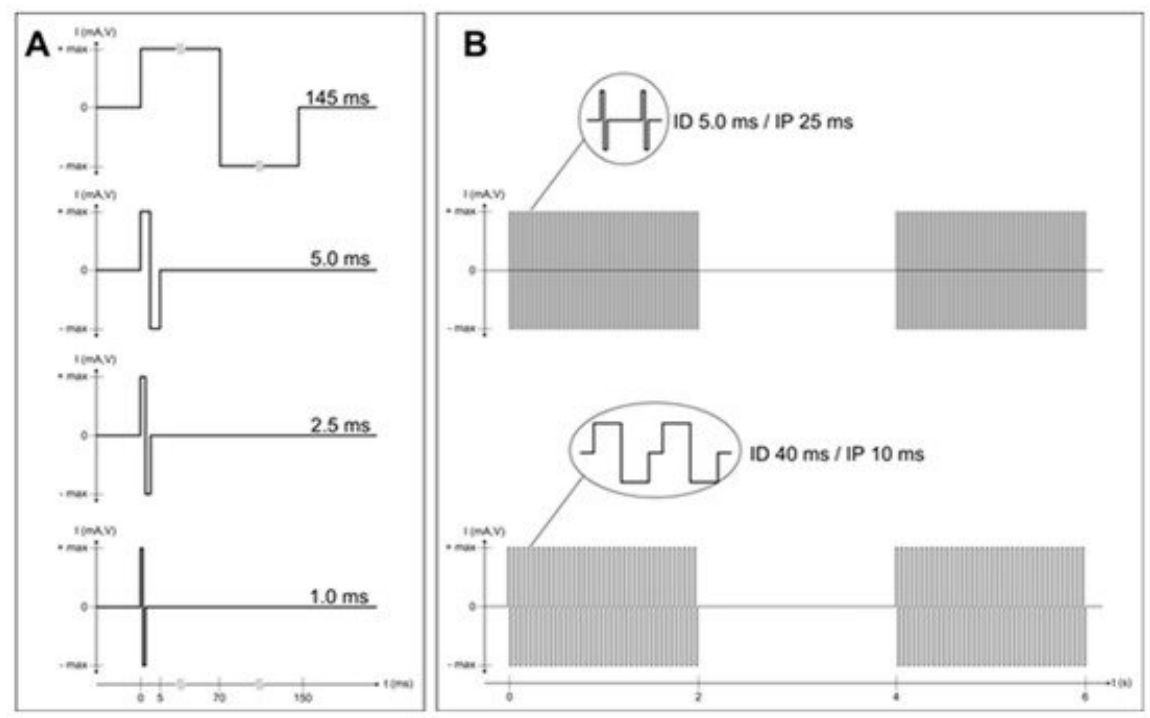

Figure 1. Complete and permanent denervation of quadriceps muscles has been confirmed by test electrical stimulation before and after two years of hbFES. Panel $(\mathbf{A})$ shows the single twitch characteristics of the electrical stimulation test at the enrolment of SCI patients in the EU RISE Project. Normal innervated muscles respond at 1.0 ms twitch stimulation. Denervated muscles tested days or weeks after SCI may respond to $2.5 \mathrm{~ms}$ or $5.0 \mathrm{~ms}$ twitch stimulation. Patients with complete muscle denervation tested 12 or more months after SCI may only respond very weakly to $145 \mathrm{~ms}$ long impulses. Three to five years after SCl, muscles seem to be unexcitable even using electrical stimuli longer than 150 ms, but after several (4-12) weeks of hbFES Vienna training, permanent denervated muscles recover contractility. During the first months of hbFES training the denervated myofibers respond to shorter and shorter test stimuli [21], but even the best responder SCI patients never return able to respond to test stimuli shorter than $40 \mathrm{~ms}$ (panel (B)), not only with macroscopic contractions, but also with minimal contractions checked by ultrasound analysis, i.e., by functional echomyography.

\section{3. hbFES of Permanent Denervated muscles by Surface Electrical Stimulation}

Two large electrodes (each $200 \mathrm{~cm} 2$ ) were fixed on the skin of the ventral aspect of the thigh fully covering the quadriceps muscles. The proximal electrode was near the inguinal fold, the distal near the knee with a minimal void in between. The electrical stimulation was applied to skin and muscles by a purpose developed stimulator and anatomically shaped large electrodes. They are all commercially available thanks to the generosity of Schuhfried Company, Vienna, Austria (see at http://schuhfriedmed.at/stimulette-en/stimulette-den2x-) [22]. Electrical stimulation 
protocol started three weeks after skin biopsy, both before and after the two years of hbFES [21]. The progressive hbFES management was personalized to the functional characteristics of the denervated muscles, i.e., mainly to SCI timespan at enrollment, never earlier than eight months from SCI. Usually, it started with 150 ms long single electrical impulses, when denervation time was longer than two years. Patients were provided for free with stimulators and electrodes to perform hbFES for five days every week. During early training, the two large conductive-polyurethane electrodes were fixed on the thigh, uby wetsponge-cloth fixed by elastic cuffs. As the skin adapted to the needed high currents, gel was placed under the polyurethane electrodes to attain minimal impedance. The electrodes were flexible enough to provide homogeneous current distribution. The training strategy consisted of four stimulation programs. At the beginning, they were biphasic stimulation impulses of long duration (120-150 ms, 60-75 ms per phase) and high intensity (up to $\pm 80 \mathrm{~V}$ and up to $\pm 250 \mathrm{~mA}$ ). The subjects underwent clinical assessment and electrostimulated knee torque test every three months by physiatrists (Physical Medicine and Rehabilitation Physicians), who progressively modified the parameters and the training protocol according to the improvements of excitability and contractility of the thigh muscles. After the early phase of hbFES, the routine daily training consisted of twitch and tetanic stimulation patterns in sessions lasting 30 min for gluteus, thigh, and lower leg muscles on right and left legs [21]. The impulse duration was progressively shortened according to the improving excitability and contractility of the muscles. When the denervated muscles recovered the ability to respond with sustained contractions to burst of stimuli (40 ms impulse duration, $10 \mathrm{~ms}$ pause $=20 \mathrm{~Hz}$ frequency), the hbFES protocol was changed to repetitive tetanic stimulation. These tetanic contractions further improve force and mass of quadriceps muscle, up to the ability to allow for standing up and stepping-in-place training [21]. This functional adjusted strategy (see Figure 2) is mandatory to obtain the clinically significant increase in quality and quantity of the thigh muscles (demonstrated in Figures 3 and 4) and the remodeling of epidermal layers of the skin [24][25][26]. Notice that Figure 4 demonstrates that the anti-atrophy effects of hbFES applied to the quadriceps muscle is extended to the non targeted hamstring muscles.

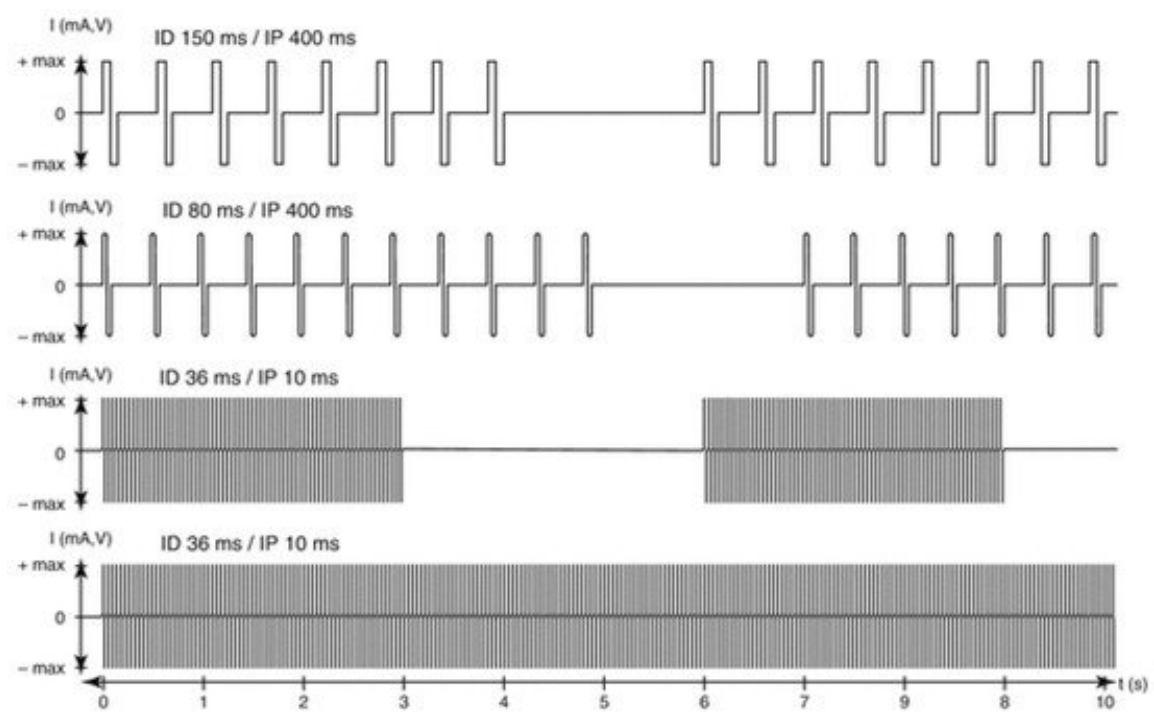

Figure 2. Parameters for progressive hbFES strategy of long-term permanently denervated human muscles. The progressive training starts, usually more than nine months after SCI, with bursts of stimulation duration (SD) of $4 \mathrm{~s}$ and a stimulation pause (SP) of $2 \mathrm{~s}$. Notice that the burst contains impulses with an impulse duration (ID) of $150 \mathrm{~ms}$ and an impulse pause (IP) of $400 \mathrm{~ms}$ to activate severely atrophic muscle fibers, able to respond only with twitch contractions. This first period of conditioning last usually 3-5 months, but could be shortened, if the hbFES starts earlier than six months from SCI. When at successive follow-up the muscle presents higher excitability and stronger twitches, the ID can be reduced to $80 \mathrm{~ms}$ and SD increased to $5 \mathrm{~s}$ for another four months. When in the following follow-up test stimulation shows that the muscle respond to ID of about $40 \mathrm{~ms}$, the next training phase implement tetanic burst stimulation of 3 s SD and 3 s SP with impulses of $40 \mathrm{~ms}$ ID and $10 \mathrm{~ms}$ IP (20 Hz frequency). The induced tetanic contractions are potent trophic factors that increase muscle mass, density and diameter of muscle fiber and then force, inducing in sitting position leg extensions without and then with additional weights on the ankles of the patients (progressive resistance training). When a good muscle condition is achieved, which depends not only to the training intensity but also to the time span of denervation, standing, stepping-in-place, and walking exercises can be performed using continuous stimulation (controlled by an external switch) with $40 \mathrm{~ms}$ ID and $10 \mathrm{~ms}$ IP. 


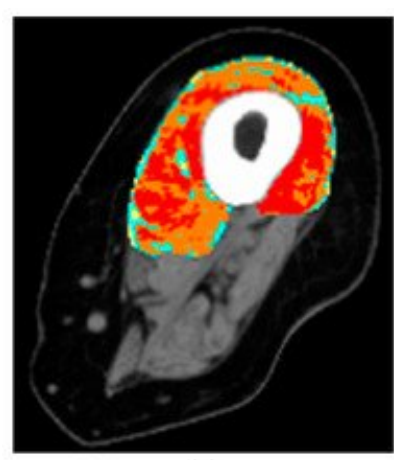

(a)

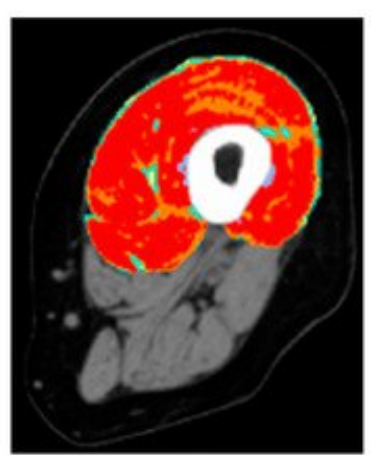

(b)

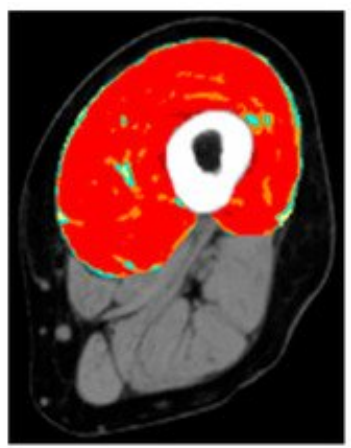

(c)

Figure 3. Colored cross-sectional area of the quadriceps muscle before and after one or two years of hbFES. In a 49year-old female patient, 2.5 years after denervation, at enrollment (a), after one year (b), and after two years (c) hbFES. The increase of muscle bulk, as shown by color CT scan analyses, is clearly present after one year of hbFES and continues during the second year of training. The muscle recovery is present also in the hamstrings, in particular after two years of hbFES. Color code for the quadriceps muscle: red = normal muscle, orange = atrophic muscle, blue = connective tissue, yellow $=$ intramuscular fat.
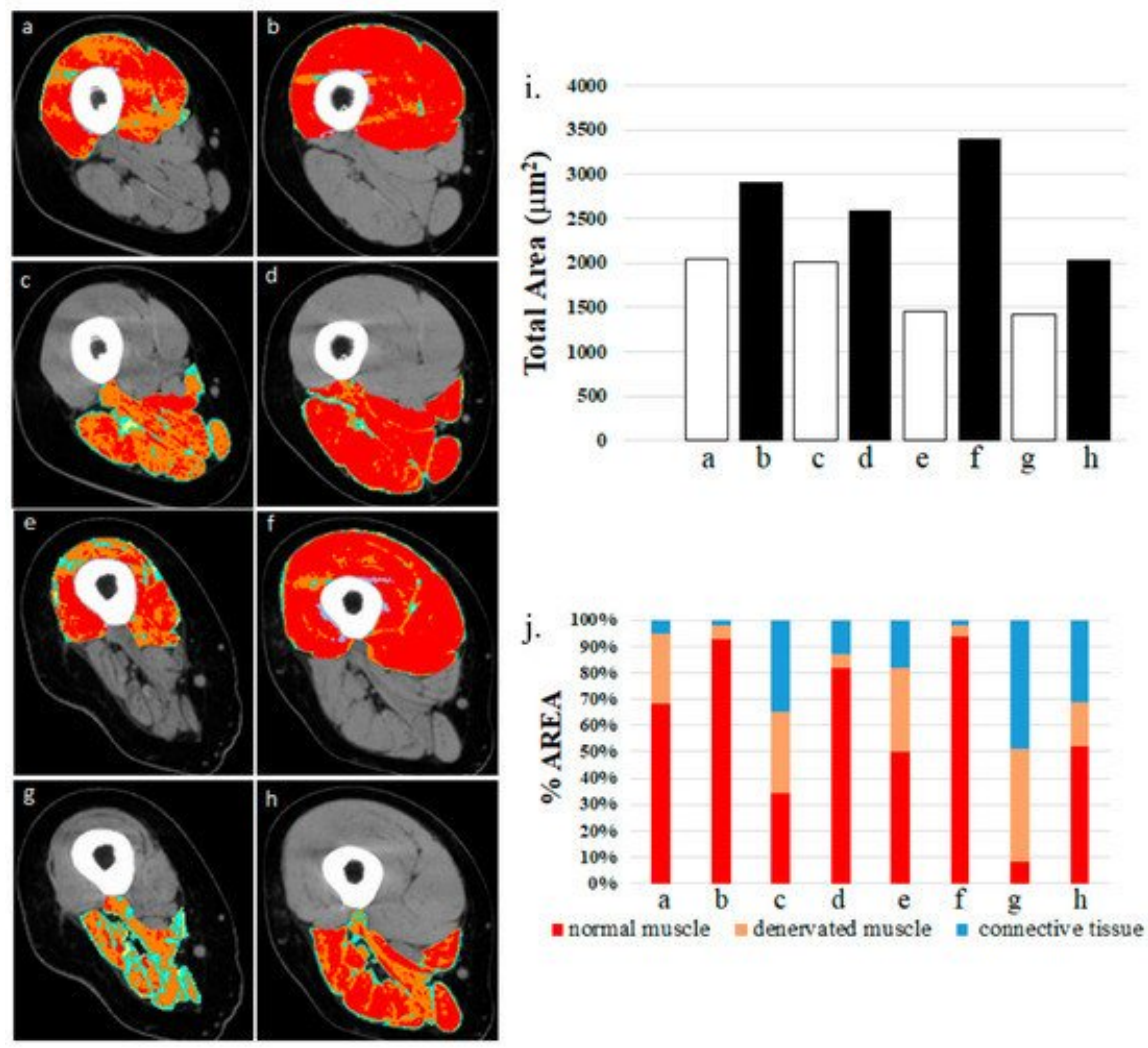

Figure 4. Denervated quadriceps and hamstring muscles respond to hbFES years after SCI. Patient A: a) quadriceps muscle at one year post-SCI, no hbFES; (b) muscle in panel "a" after two years of hbFES. Patient B: $\mathbf{c}$ ) hamstring muscle at one year post-SCI, no hbFES; (d) muscle in panel "c" after two years of hbFES; e) Patient C: quadriceps muscle at three years post-SCI, no hbFES; (f) muscle in panel "e" after two years of hbFES. Patient D: $\mathbf{g}$ ) hamstring muscle at three years post-SCI, no hbFES; (h) muscle in panel "g" after two years of hbFES (i). Total area of muscles $\mathrm{a}-\mathrm{h}(\mathrm{j})$. Quantitative computed densitometric analyses of tomography, muscles a-h. (Reproduced with permission from $[23]$.

\subsection{The recovery of Permanent Denervated Muscles from atrophy and degeneration by hbFES is not secondary to a reinnervation process. Evidence by Test Stimulation}

Complete and permanent denervation of quadriceps muscles has been confirmed by test electrical stimulation before and after two years of hbFES. In Figure 1, Panel (a) shows the single twitch characteristics of the electrical stimulation test at the enrolment of SCI patients in the EU RISE Project. Normal innervated muscles respond at $1.0 \mathrm{~ms}$ twitch stimulation. Denervated muscles tested days or weeks after SCI may respond to $2.5 \mathrm{~ms}$ or 5.0 ms twitch stimulation. 
Patients with complete muscle denervation tested 12 or more months after SCI may only respond very weakly to 145 ms long impulses. Three to five years after SCI, muscles seem to be unexcitable even using electrical stimuli longer than 150 ms, but after several (4-12) weeks of hbFES Vienna training, permanent denervated muscles recover contractility. During the first months of hbFES training the denervated myofibers respond to shorter and shorter test stimuli [21], but even the best responder SCI patients never return able to respond to test stimuli shorter than $40 \mathrm{~ms}$ (panel (b), not only with macroscopic contractions, but also with minimal contractions checked by ultrasound analysis, i.e., by functional echomyography.

\subsection{The recovery of Permanent Denervated Muscles from atrophy and degeneration by hbFES is not secondary to a reinnervation process. Evidence by Quantitative Muscle Color Computed Tomography in compliant and non-compliant patients}

We determined the gross anatomy of the thigh muscles and the extent of their atrophy/degeneration using quantitative muscle color computed tomography (QMC-CT) [21][27][28]. QMC-CT uses the Hounsfield units for tissue characterization. Soft tissues were quantitated as subcutaneous fat, intramuscular fat, low-density muscle, normal muscle, and fibrous-dense connective tissue. To measure muscle degeneration, the pixels within defined ranges of Hounsfield units were colored (red for normal muscle, yellow for intramuscular adipose tissue, green and blue for loose and fibrous connective tissues, respectively) [29]. Quadriceps muscles degenerate dramatically as a consequence of long-term denervation in SCI patients. These muscles become thinner and their characteristic single bellies are not easily recognisable, except in the rectus femoris (RF). Therefore, to assess muscle density during and after hbFES, RF became the target muscle to quantify the expected morphological changes. Thus, after the patients were subjected to CT scans of the thigh muscles, the datasets were segmented via the MIMICS software, to gather information about their density in $\mathrm{HU}$, as well as to build three dimensional (3D) models in order to analyse the anatomical evolution during and after hbFES [30][31][32][33][34][35][36][37][38].

\subsection{The recovery of Permanent Denervated Muscles from atrophy and degeneration by hbFES is not secondary to a reinnervation process: Evidence by 3D gross anatomy and muscle density of the Rectus Femoris (RF)}

Figure 5.A shows the evolution of muscle density throughout the years, representing a 3D model of the right femur (in white) and rectus femoris (in red), for every year of the study [39]. The first year is denoted as the year 0 , immediately before the FES protocol started four years after SCI. Then, the patient was measured regularly during 5 years since the beginning of the FES, and one last time 4 years after stopping FES. Compared to the year 0, an evolution in the muscle shape is noticeable after the beginning of the FES. Certainly, the muscle size is increased than the previous year. However, after the FES protocol stopped, the dataset shows a thinner/smaller muscle, which resembles the one pre-FES. The difference throughout the years is also noticeable in the data plotted in Figure 4.B, where each dataset plots the average $\mathrm{HU}$ value of the muscle, as well as their standard deviation. For each year after year 0 , the $\mathrm{HU}$ value increases until dropping considerably after the FES was stopped. These results confirm what is seen in figure 4.A, where a denser muscle appears while being stimulated but that size reduced when the stimulation stops. 


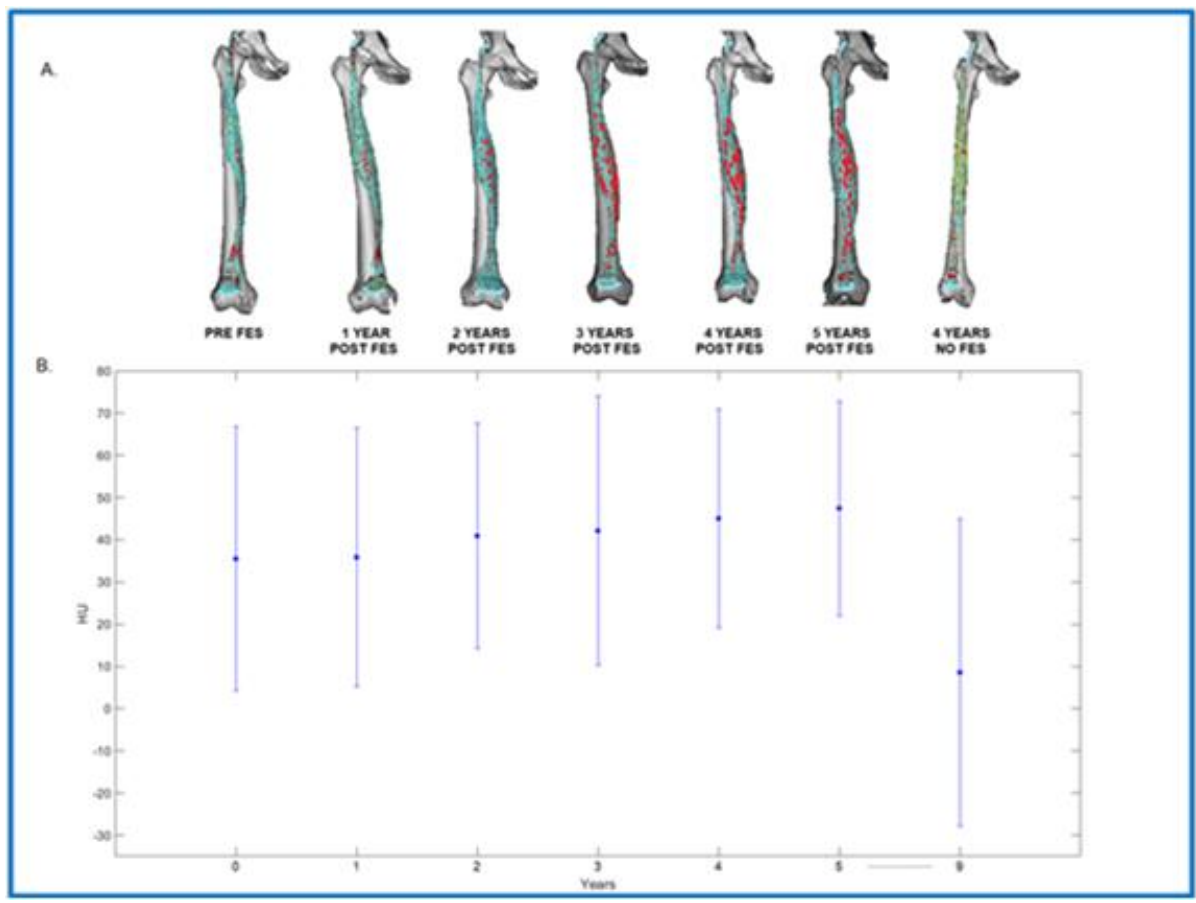

Figure 5. (A) Muscle density of rectus femoris throughout the years. (B) Plots of the average HU values of the RF muscle and their standard deviation.

Table 1 below is a summary of all the results of the patient's datasets throughout the years, with first the average and standard deviation of $\mathrm{HU}$, that are plotted in figure 4.B. Then the muscle volume is presented in $\mathrm{mm} 3$ as well as its relative variation in \% compared to the year 0 . We can see that every year post FES (apart from year 2, which still has an increased volume than year 0 ), the volume variation is increased. When the FES stopped, the results show a decrease of volume of $30,5 \%$ compared to the year pre-FES.

\begin{tabular}{|c|c|c|c|c|c|c|c|}
\hline Year FES (years of SCI) & $0(4)$ & $1(5)$ & $2(6)$ & $3(7)$ & $4[8]$ & $5(9)$ & +4_noFES (13) \\
\hline HU average value & 35,51 & 35,87 & 40,93 & 42,14 & 45,08 & 47,47 & 8,59 \\
\hline HU STD & 31,31 & 30,62 & 26,73 & 31,92 & 25,84 & 25,44 & 36,36 \\
\hline Mask Volume (mm3) & 89028 & 123627 & 99577 & 134496 & 137015 & 145068 & 61917 \\
\hline Volume variation (\%) & 0 & 38,9 & 11,8 & 51,1 & 53,9 & 62,9 & $-30,5$ \\
\hline Fat $\%$ & 7,81 & 8,41 & 0,23 & 6,04 & 0 & 0 & 35,26 \\
\hline Connective \% & 47,85 & 46,46 & 52,84 & 40,33 & 41,96 & 36,98 & 45,55 \\
\hline Muscle \% & 44,35 & 45,13 & 46,87 & 53,62 & 58,04 & 63,01 & 19,2 \\
\hline Fat variation (\%) & 0,00 & 0,60 & $-7,58$ & $-1,77$ & $-7,81$ & $-7,81$ & 27,45 \\
\hline Connective variation (\%) & 0,00 & $-1,39$ & 4,99 & $-7,52$ & $-5,89$ & $-10,87$ & $-2,30$ \\
\hline Muscle variation (\%) & 0,00 & 0,78 & 2,52 & 9,27 & 13,69 & 18,66 & $-25,15$ \\
\hline
\end{tabular}

Finally, the last rows show the absolute values of fat, connective tissue and muscle percentages, as well as their percentage variation compared to year 0 . To separate each component, we chose $\mathrm{HU}$ intervals: Fat: -200 to -10 HU, Connective tissue: -9 to $40 \mathrm{HU}$, Muscle: 41 to $200 \mathrm{HU}$. If we observe the relative variation, we can see that the fat decreases almost steadily after the stimulation, with around $7 \%$ less than pre-FES. When the FES stops, the fat percentage rises to $27 \%$ more than before the FES, representing $35 \%$ of the organ. The opposite behavior is observed for the muscle tissue. Its percentage variation is highly increased throughout the years post FES, with $18,66 \%$ more after 5 years. Then, when the electro-stimulation stops, it drops to $-25 \%$ compared to year 0 , representing only $19 \%$ of the organ.

In summary, Figure 5 and Table 1 confirm that in a compliant patient permanent denervated muscles respond to hbFES for several years, even several years after $\mathrm{SCl}$, and that the positive results disappear if the patient is no longer compliant. Thus, the positive effects were related to the direct trophic effects of hbFES-induced muscle contractions, not to muscle reinnervation. 


\section{Byproducts}

\subsection{Neuromuscular Electrical Stimulation Appropriate for Elderly People}

Standing on the results of the EU RISE Program, the Colleagues of Vienna, Austria designed and realized stimulators for home-based neuromuscular electrical stimulation appropriate for elderly people. As described in Kern et al. [40], elderly persons performed neuromuscular electrical stimulation training at home. Results demonstrated that when training for nine weeks (two times a week $3 \times 10$ min for each session), neuromuscular electrical stimulation for elderly persons is safe and effective. All subjects achieved neuromuscular electrical stimulation-induced full knee extension. The outcomes included a significant increase in muscle strength and an increase in the number and size of fast myofibers, that are the first to respond to electrical stimulation and whose content and size are correlated to muscle strength. In muscle biopsies, Pax7- and NCAM-positive muscle satellite cells were also increased in the absence of muscle damage and cellular inflammation [41][42][43][44][45][46][47][48][49][50][51]. Finally, there are many applications of in-home or in-hospital functional electrical stimulation managements of disuse muscle atrophy related to organ diseases, from chronic cardiovascular failures to functional electrostimulation cycling in SCI [52][53][54][55][56].

\subsection{Machine Learning Predictive Systems}

A further byproduct is a predictive system based on radiodensitometric distributions from mid-thigh CT images. Muscle deterioration in elderly individuals is commonly characterized by loss of muscle strength and replacement of lean tissue mass with inter- and intra-muscular adipose tissue. These phenomena are independent mortality risks in aging individuals. The incidence of muscle deterioration in aging, commonly referred to as sarcopenia, significantly affects the quality of life and physical activity of aging individuals [57]. Machine learning (ML) algorithms, are becoming increasingly used in healthcare data applications. The increased availability of healthcare data and the continued development of big data analytics methods has driven the success of ML modelling in many quantitative fields, such as medical image processing or predictive system development, as well as other specialties such as neurology, cardiology, and oncology [58][59][60][61][62]. Mid-thigh computed tomography (CT) images from the Age, Gene/Environment Susceptibility (AGES) dataset have been used to quantitatively characterize subject-specific changes in soft tissue using a novel method known as non-linear trimodal regression analysis (NTRA) [63]. The NTRA method works by generating soft tissue regression profiles described by 11 unique NTRA model parameters. The utility of these parameters in quantifying differences in fat, lean muscle, and loose connective tissue was first explored in comparing young, aging, and pathological subjects. Results from this work illustrated the sensitivity of NTRA parameters to changes in soft tissue and suggested the employment of this method in the context of a larger CT image database. The Age Gene/Environment Susceptibility Study (AGES-Reykjavík) is an Icelandic dataset designed to examine risk factors and gene/environment interactions in relation to disease and disability in aging people. This dataset was assembled using 3152 volunteers from 66-92 years of age analyzed at two time points separated by five years. The AGES-Reykjavík dataset thereby presents a unique opportunity to employ big data analytics methods such as $\mathrm{ML}$ modelling ${ }^{[64]}$. As $\mathrm{ML}$ algorithms have illustrated strong predictive value in the regression of body mass index (BMI) and isometric leg strength (ISO), the study sought to demonstrate their prediction using NTRA parameters obtained from CT mid-femur cross-sections in the AGES-Reykjavík dataset. Results from this work further consolidate the predictive power of NTRA using BMI and ISO as test parameters. The method is useful in prediction studies of mobility and cardiocirculatory diseases [27][65]. Further investigations of the correlations between these parameters and the consequent risks could further expand the field of translational myology to sarcopenic muscle degeneration and its downstream effects on the health of the elderly.

\section{Conclusions}

We demonstrated the long-term clinical value of co-activating thigh muscles through hbFES strategy using high currents and large surface electrodes. This Vienna Strategy is able to reverse, at clinically relevant levels, the adverse effects of $\mathrm{SCl}$, even in the worst-case scenario of permanent lower motor neuron denervation of leg muscles in complete conus and cauda equina. Continued regularly, hbFES for denervated, degenerating muscles helps to maintain healthier leg muscles and skin, reducing the risks of life-threatening $\mathrm{SCl}$ complications.

We hope that international physiatrists will collaborate in new independent multi-center trials for denervated, 
degenerating muscles recovery by hbFES. Involvement of physiatrists and physiotherapists will be helpful in other centers worldwide, including where there is already interest, such as in Brasil and Russia [66][67][68][69][70], to offer to these relatively rare SCI patients with permanent complete damage of conus and cauda equina the opportunities they deserve.

\section{References}

1. Marcell, T. J.; Sarcopenia: causes, consequences and preventions. . J Gerontol A Biol Sci Med Sci 2003, 58, M911-M916, doi.org/10.1093/gerona/58.10.M911.

2. Gava, P.; Giuriati, W.; Ravara, B. Gender difference of aging performance decay rate in normalized Masters World Records of Athletics: Much less than expected. Eur. J. Transl. Myol. 2020, 30, 8869, doi:10.4081/ejtm.2019.8869. eCollection 7 April. 2020.

3. Spillman, B.C.; Lubitz, J. The effect of longevity on spending for acute and long-term care. New Engl. J. Med. 2000, 342, 1409-1415, doi:10.1056/NEJM200005113421906.

4. Coletti, D. Chemotherapy-induced muscle wasting: An update. Eur. J. Transl. Myol. 2018, 28, 7587, doi:10.4081/ejtm.2018.7587.

5. Chandrasekaran, S.; Davis, J.; Bersch, I.; Goldberg, G.; Gorgey, A.S. Electrical stimulation and denervated muscles after spinal cord injury. Neural Regen. Res. 2020, 15, 1397-1407, doi:10.4103/1673-5374.274326.

6. Hopkins, R.O.; Mitchell, L.; Thomsen, G.E.; Schafer, M.; Link, M.; Brown, S.M. Implementing a mobility program to minimize postintensive care syndrome. AACN Adv. Crit. Care 2016, 27, 187-203, doi:10.4037/aacnacc2016244.

7. Bolotta, A.; Filardo, G.; Abruzzo, P.M.; Astolfi, A.; De Sanctis, P.; Di Martino, A.; Hofer, C.; Indio, V.; Kern, H.; Löfler, S.; et al. Skeletal Muscle Gene Expression in Long-Term Endurance and Resistance Trained Elderly. Int. J. Mol. Sci. 2020, 21, E3988, doi:10.3390/ijms21113988.

8. Šarabon, N.; Smajla, D.; Kozinc, Ž.; Kern, H. Speed-power based training in the elderly and its potential for daily movement function enhancement. Eur. J. Transl. Myol. 2020, 30, 8898, doi:10.4081/ejtm.2019.8898.

9. Choi, Y.; Cho, J.; No, M.H.; Heo, J.W.; Cho, E.J.; Chang, E.; Park, D.H.; Kang, J.H.; Kwak, H.B. Re-Setting the Circadian Clock Using Exercise against Sarcopenia. Int. J. Mol. Sci. 2020, 21, 3106, doi:10.3390/ijms21093106.

10. Jones, S.; Man, W.D.; Gao, W.; Higginson, I.J.; Wilcock, A.; Maddocks, M. Neuromuscular electrical stimulation for muscle weakness in adults with advanced disease. Cochrane Database Syst. Rev. 2016, 10, D009419, doi:10.1002/14651858.CD009419.pub3.

11. Mastryukova, V.; Arnold, D.; Güllmar, D.; Guntinas-Lichius, O.; Volk, G.F. Can MRI quantify the volume changes of denervated facial muscles? Eur. J. Transl. Myol. 2020, 30, 8918, doi:10.4081/ejtm.2019.8918.

12. Ades, P.A.; Keteyian, S.J.; Wright, J.S.; Hamm, L.F.; Lui, K.; Newlin, K.; Shepard, D.S.; Thomas, R.J. Increasing Cardiac Rehabilitation Participation From 20\% to 70\%: A Road Map From the Million Hearts Cardiac Rehabilitation Collaborative. Mayo Clin. Proc. 2017, 92, 234-242, doi:10.1016/j.mayocp.2016.10.014.

13. Vorona, S.; Sabatini, U.; Al-Maqbali, S.; Bertoni, M.; Dres, M.; Bissett, B.; Van Haren, F.; Martin, A.D.; Urrea, C.; Brace, D.; et al. Inspiratory Muscle Rehabilitation in Critically III Adults: A Systematic Review and Meta-Analysis. Ann. Am. Thorac. Soc. 2018, 15, 735-744, doi:10.1513/AnnalsATS.201712-9610C.

14. Etoom, M. Comments on: Influence of transcutaneous electrical nerve stimulation on spasticity, balance, and walking speed in stroke patients: A systematic review and meta-analysis. J. Rehabil. Med. 2018, 50, 94, doi:10.2340/16501977-2303.

15. Kiper, P.; Turolla, A. Updates and comments on: Influence of transcutaneous electrical nerve stimulation on spasticity, balance, and walking speed in stroke patients: A systematic review and meta-analysis. J. Rehabil. Med. 2019, 51, 317-318, doi:10.2340/16501977-2538.

16. Bersch, I.; Tesini, S.; Bersch, U.; Frotzler, A. Functional electrical stimulation in spinal cord injury: Clinical evidence versus daily practice. Artif. Organs 2015, 39, 849-854, doi:10.1111/aor.12618.

17. Lin, S.; Sun, Q.; Wang, H.; Xie, G. Influence of transcutaneous electrical nerve stimulation on spasticity, balance, and walking speed in stroke patients: A systematic review and meta-analysis. J. Rehabil. Med. 2018, 50, 3-7, doi:10.2340/16501977-2266.

18. Burgess, L.C.; Immins, T.; Swain, I.; Wainwright, T.W. Effectiveness of neuromuscular electrical stimulation for reducing oedema: A systematic review. J. Rehabil. Med. 2019, 51, 237-243, doi:10.2340/16501977-2529.

19. Laubacher, M.; Aksoez, E.A.; Brust, A.K.; Baumberger, M.; Riener, R.; Binder-Macleod, S.; Hunt, K.J. Stimulation of paralysed quadriceps muscles with sequentially and spatially distributed electrodes during dynamic knee extension. J. Neuroeng. Rehabil. 2019, 16, 5, doi:10.1186/s12984-018-0471-y.

20. Angeli, C.A.; Boakye, M.; Morton, R.A.; Vogt, J.; Benton, K.; Chen, Y.; Ferreira, C.K.; Harkema, S.J. Recovery of Over-Ground Walking after Chronic Motor Complete Spinal Cord Injury. New Engl. J. Med. 2018, 379, 1244-1250, doi:10.1056/NEJMoa1803588.

21. Kern, H.; Carraro, U.; Adami, N.; Biral, D.; Hofer, C.; Forstner, C.; Mödlin, M.; Vogelauer, M.; Pond, A.; Boncompagni, S.; et al. Home-based functional electrical stimulation rescues permanently denervated muscles in paraplegic patients with complete lower motor neuron lesion. Neurorehabil. Neural Repair 2010, 24, 709-721, doi:10.1177/1545968310366129.

22. Schuhfried Medizintechnik. Available online: https://schuhfriedmed.at/elektro-und-reizstromtherapie/ (accessed on 16 June 2020).

23. Carraro, U.; Kern, H.; Albertin, G.; Masiero, S.; Pond, A.L.; Gargiulo, P. Functional Electrical Stimulation of Permanently Denervated Muscles. Bull. Rehabil. Med. 2020, 97, 130-136, doi:10.38025/2078-1962-2020-97-3-130-136. 
24. Ravara, B.; Hofer, C.; Kern, H.; Guidolin, D.; Porzionato, A.; De Caro, R.; Albertin, G. Dermal papillae flattening of thigh skin in Conus Cauda Syndrome. Eur. J. Transl. Myol. 2018, 28, 7914, doi:10.4081/ejtm.2018.7914.

25. Albertin, G.; Hofer, C.; Zampieri, S.; Vogelauer, M.; Löfler, S.; Ravara, B.; Guidolin, D.; Fede, C.; Incendi, D.; Porzionato, A.; De Caro, R.; Baba, A.; Marcante, A.; Piccione, F.; Gargiulo, P.; Pond, A.; Carraro, U.; Kern, H. In complete SCI patients, long-term functional electrical stimulation of permanent denervated muscles increases epidermis thickness. Neurol. Res. 2018 Apr;40(4):277282. doi: $10.1080 / 01616412.2018 .1436877$.

26. Albertin, G.; Ravara, B.; Kern, H.; Hofer, C.; Loefler, S.; Jurecka, W.; Guidolin, D.; Rambaldo, A.; Porzionato, A.; De Caro, R.; et al. Two-years of home based functional electrical stimulation recovers epidermis from atrophy and flattening after years of complete Conus-Cauda Syndrome. Medicine (Baltimore) 2019, 98, e18509, doi:10.1097/MD.0000000000018509.

27. Ricciardi, C.; Edmunds, K.J.; Recenti, M.; Sigurdsson, S.; Gudnason, V.; Carraro, U.; Gargiulo, P. Assessing cardiovascular risks from a mid-thigh CT image: A tree-based machine learning approach using radiodensitometric distributions. Sci. Rep. 2020, 10, 2863, doi:10.1038/s41598-020-59873-9.

28. Gislason, M.K.; Ingvarsson, P.; Gargiulo, P.; Yngvason, S.; Guð̃mundsdóttir, V.; Knútsdóttir, S.; Helgason, p. Finite Element Modelling of the Femur Bone of a Subject Suffering from Motor Neuron Lesion Subjected to Electrical Stimulation. Eur. J. Transl. Myol. 2015, 24, 2187, doi:10.4081/ejtm.2014.2187.

29. Edmunds, K.J.; Gíslason, M.K.; Arnadottir, I.D.; Marcante, A.; Piccione, F.; Gargiulo, P. Quantitative Computed Tomography and Image Analysis for Advanced Muscle Assessment. Eur. J. Transl. Myol. 2016, 26, 6015, doi:10.4081/ejtm.2016.6015.

30. Kern, H.; Boncompagni, S.; Rossini, K.; Mayr, W.; Fanò, G.; Zanin, M.E.; Podhorska-Okolow, M.; Protasi, F.; Carraro, U. Long-term denervation in humans causes degeneration of both contractile and excitation- contraction coupling apparatus, wich is reversibile by functional electrical stimulation (FES). A role for myofiber regeneration? J. Neuropathol. Exp. Neurol. 2004, 63, 919-931, doi:10.1093/jnen/63.9.919.

31. Boncompagni, S.; Pozzer, D.; Viscomi, C.; Ferreiro, A.; Zito, E. Physical and Functional Cross Talk Between Endo-Sarcoplasmic Reticulum and Mitochondria in Skeletal Muscle. Antioxid. Redox. Signal. 2020, 32, 873-883, doi:10.1089/ars.2019.7934.

32. Edmunds, K.J.; Gargiulo, P. Imaging Approaches in Functional Assessment of Implantable Myogenic Biomaterials and Engineered Muscle Tissue. Eur. J. Transl. Myol. 2015, 25, 4847, doi:10.4081/ejtm.2015.4847. eCollection 11 March 2015. Review.

33. Kern, H.; Carraro, U.; Adami, N.; Hofer, C.; Loefler, S.; Vogelauer, M.; Mayr, W.; Rupp, R.; Zampieri, S. One year of home-based daily FES in complete lower motor neuron paraplegia: Recovery of tetanic contractility drives the structural improvements of denervated muscle. Neurol. Res. 2010, 32, 5-12, doi:10.1179/174313209X385644.

34. Zanato, R.; Martino, L.; Carraro, U.; Kern, H.; Rossato, E.; Masiero, S.; Stramare, R. Functional Echomyography: Thickness, ecogenicity, contraction and perfusion of the LMN denervated human muscle before and during h-bFES. Eur. J. Transl. Myol. 2010, 20, 33-40.

35. Yang, K.-C.; Liao, Y.-Y.; Chang, K.-V.; Huang, K.-C.; Han, D.-S. The Quantitative Skeletal Muscle Ultrasonography in Elderly with Dynapenia but Not Sarcopenia Using Texture Analysis. Diagnostics 2020, 10, 400, doi:10.3390/diagnostics10060400.

36. Romero-Morales, M.; Martín-Llantino, P.; Calvo-Lobo, C.; San-Antolín, M.; López-López, D.; Blanco-Morales, M.; Rodríguez-Sanz, D. Ultrasound Imaging of the Abdominal Wall and Trunk Muscles in Patients with Achilles Tendinopathy versus Healthy Participants. Diagnostics 2020, 10, 17, doi:10.3390/diagnostics10010017.

37. Hitzig, S.L.; Eng, J.J.; Miller, W.C.; Sakakibara, B.M. An evidence-based review of aging of the body systems following spinal cord injury. Spinal Cord 2011, 49, 684-701, doi:10.1038/sc.2010.178.

38. Giangregorio, L.; McCartney, N. Bone Loss and Muscle Atrophy in Spinal Cord Injury: Epidemiology, Fracture Prediction, and Rehabilitation Strategies. J. Spinal Cord Med. 2006, 29, 489-500, doi:10.1080/10790268.2006.11753898.

39. Gargiulo, P.; Helgason, T.; Ingvarsson, P.; Mayr, W.; Kern, H.; Carraro, U. Medical image analysis and 3-d modeling to quantify changes and functional restoration in denervated muscle undergoing electrical stimulation treatment. Hum-Cent Comput Info. 2012, 2, 10, doi.org/10.1186/2192-1962-2-10

40. Kern, H.; Barberi, L.; Löfler, S.; Sbardella, S.; Burggraf, S.; Fruhmann, H.; Carraro, U.; Mosole, S.; Sarabon, N.; Vogelauer, M.; et al. Electrical stimulation counteracts muscle decline in seniors. Front. Aging Neurosci. 2014, 6, 189, doi:10.3389/fnagi.2014.00189.

41. Mayr, W. Neuromuscular Electrical Stimulation for Mobility Support of Elderly. Eur. J. Transl. Myol. 2015, 25, 263-268, doi:10.4081/ejtm.2015.5605.

42. Protasi, F. Mitochondria Association to Calcium Release Units is Controlled by Age and Muscle Activity. Eur. J. Transl. Myol. 2015, 25, 257-262, doi:10.4081/ejtm.2015.5604.

43. Sarabon, N.; Löfler, S.; Hosszu, G.; Hofer, C. Mobility Test Protocols for the Elderly: A Methodological Note. Eur. J. Transl. Myol. 2015, 25, 253-256, doi:10.4081/ejtm.2015.5385.

44. Cvecka, J.; Tirpakova, V.; Sedliak, M.; Kern, H.; Mayr, W.; Hamar, D. Physical Activity in Elderly. Eur. J. Transl. Myol. 2015, 25, 249252, doi:10.4081/ejtm.2015.5280.

45. Forcina, L.; Cosentino, M.; Musarò, A. Mechanisms Regulating Muscle Regeneration: Insights into the Interrelated and TimeDependent Phases of Tissue Healing. Cells 2020, 9, 1297, doi:10.3390/cells9051297.

46. Sajer, S.; Guardiero, G.S.; Scicchitano, B.M. Myokines in Home-Based Functional Electrical Stimulation-Induced Recovery of Skeletal Muscle in Elderly and Permanent Denervation. Eur. J. Transl. Myol. 2018, 28, 7905, doi:10.4081/ejtm.2018.7905. 
47. Scicchitano, B.M.; Sica, G.; Musarò, A. Stem Cells and Tissue Niche: Two Faces of the Same Coin of Muscle Regeneration. Eur. J. Transl. Myol. 2016, 26, 6125, doi:10.4081/ejtm.2016.6125.

48. Barberi, L.; Scicchitano, B.M.; Musaro, A. Molecular and Cellular Mechanisms of Muscle Aging and Sarcopenia and Effects of Electrical Stimulation in Seniors. Eur. J. Transl. Myol. 2015, 25, 231-236, doi:10.4081/ejtm.2015.5227.

49. Taylor, M.J.; Fornusek, C.; Ruys, A.J. Reporting for Duty: The duty cycle in Functional Electrical Stimulation research. Part I: Critical commentaries of the literature. Eur. J. Transl. Myol. 2018, 258 7732, doi:10.4081/ejtm.2018.7732.

50. Taylor, M.J.; Fornusek, C.; Ruys, A.J. The duty cycle in Functional Electrical Stimulation research. Part II: Duty cycle multiplicity and domain reporting. Eur. J. Transl. Myol. 2018, 258, 7733, doi:10.4081/ejtm.2018.7733.

51. Taylor, M.J.; Schils, S.; Ruys, A.J. Home FES: An Exploratory Review. Eur. J. Transl. Myol. 2019, 29, 8285, doi:10.4081/ejtm.2019.8285.

52. Quittan, M.; Sochor, A.; Wiesinger, G.F.; Kollmitzer, J.; Sturm, B.; Pacher, R.; Mayr, W. Strength improvement of knee extensor muscles in patients with chronic heart failure by neuromuscular electrical stimulation. Artif. Organs 1999, 23, 432-435, doi:10.1046/j.1525-1594.1999.06372.x.

53. Deley, G.; Denuziller, J.; Babault, N. Functional electrical stimulation: Cardiorespiratory adaptations and applications for training in paraplegia. Sports Med. 2015, 45, 71-82, doi:10.1007/s40279-014-0250-2.

54. Braz, G.P.; Russold, M.F.; Fornusek, C.; Hamzaid, N.A.; Smith, R.M.; Davis, G.M. Cardiorespiratory and Muscle Metabolic Responses During Conventional Versus Motion Sensor-Assisted Strategies for Functional Electrical Stimulation Standing After Spinal Cord Injury. Artif. Organs 2015, 39, 855-862, doi:10.1111/aor.12619.

55. Crevenna, R.; Wolzt, M.; Fialka-Moser, V.; Keilani, M.; Nuhr, M.; Paternostro-Sluga, T.; Pacher, R.; Mayr, W.; Quittan, M. Long-term transcutaneous neuromuscular electrical stimulation in patients with bipolar sensing implantable cardioverter defibrillators: A pilot safety study. Artif. Organs 2004, 28, 99-102, doi:10.1111/j.1525-1594.2004.40006.x.

56. Coste, C.A.; Bergeron, V.; Berkelmans, R.; Martins, E.F.; Fornusek, C.; Jetsada, A.; Hunt, K.J.; Tong, R.; Triolo, R.; Wolf, P. Comparison of strategies and performance of functional electrical stimulation cycling in spinal cord injury pilots for competition in the first ever CYBATHLON. Eur. J. TransI. Myol. 2017, 27, 7219, doi:10.4081/ejtm.2017.7219.

57. Mitchell, W.K.; Williams, J.; Atherton, P.; Larvin, M.; Lund, J.; Narici, M. Sarcopenia, dynapenia, and the impact of advancing age on human skeletal muscle size and strength; a quantitative review. Front. Physiol. 2012, 3, 260, doi:10.3389/fphys.2012.00260.

58. Rommers, N.; RÖssler, R.; Verhagen, E.; Vandecasteele, F.; Verstockt, S.; Vaeyens, R.; Lenoir, M.; D'Hondt, E.; Witvrouw, E. A Machine Learning Approach to Assess Injury Risk in Elite Youth Football Players. Med. Sci. Sports Exerc. 2020, 52, 1745-1751, doi:10.1249/MSS.0000000000002305.

59. Burgos, N.; Colliot, O. Machine learning for classification and prediction of brain diseases: Recent advances and upcoming challenges. Curr. Opin. Neurol. 2020, 33, 439-450, doi:10.1097/WCO.0000000000000838.

60. Saxby, D.J.; Killen, B.A.; Pizzolato, C.; Carty, C.P.; Diamond, L.E.; Modenese, L.; Fernandez, J.; Davico, G.; Barzan, M.; Lenton, G.; et al. Machine learning methods to support personalized neuromusculoskeletal modelling. Biomech. Model. Mechanobiol. 2020, doi:10.1007/s10237-020-01367-8.

61. Lin, A.; Dey, D. CT-based radiomics and machine learning for the prediction of myocardial ischemia: Toward increasing quantification. J. Nucl. Cardiol. 2020, doi:10.1007/s12350-020-02261-7.

62. Agne, N.A.; Tisott, C.G.; Ballester, P.; Passos, I.C.; Ferrão, Y.A. Predictors of suicide attempt in patients with obsessive-compulsive disorder: An exploratory study with machine learning analysis. Psychol. Med. 2020, 16, 1-11, doi:10.1017/S0033291720002329.

63. Recenti, M.; Ricciardi, C.; Edmunds, K.; Gislason, M.K.; Gargiulo, P. Machine learning predictive system based upon radiodensitometric distributions from mid-thigh CT images. Eur. J. Transl. Myol. 2020, 30, 8892, doi:10.4081/ejtm.2019.8892.

64. Harris, T.B.; Launer, L.J.; Eiriksdottir, G.; Kjartansson, O.; Jonsson, P.V.; Sigurdsson, G.; Thorgeirsson, G.; Aspelund, T.; Garcia, M.E.; Cotch, M.F.; et al. Age, Gene/Environment Susceptibility-Reykjavik Study: Multidisciplinary Applied Phenomics. Am. J. Epidemiol. 2007, 165, 1076-1087, doi:10.1093/aje/ kwk115.

65. Edmunds, K.J.; Petersen, H.; Hassan, M.; Yassine, S.; Olivieri, A.; Barollo, F.; Friðriksdóttir, R.; Edmunds, P.; Gíslason, M.K.; Fratini, A.; et al. Cortical recruitment and functional dynamics in postural control adaptation and habituation during vibratory proprioceptive stimulation. J. Neural. Eng. 2019, 16, 026037, doi:10.1088/1741-2552/ab0678.

66. Molchanova, E.E. Clinical efficiency of dynamic electroneurostimulation in the acute period of the ischemic stroke. Bull. Rehabil. Med. 2015, 65, 33-36 (In Russian ).

67. Evstigneeva, L.P.; Polyanskaya, T.P.; Vlasov, A.A. The role of dynamic electricneurostimulation in reducing pain and improving quality of life of patients with osteoporosis. Bull. Rehabil. Med. 2015, 67, 19-28. (In Russian)

68. Drobyshev, V.A.; Gerasimenko, O.N.; Romanovskaya, N.S.; Vlasov, A.A.; Shashukov, D.A. Effectiveness of dynamic electrical stimulation in complex treatment in acute period of ischemic stroke. Bull. Rehabil. Med. 2016, 72, 21-26. (In Russian)

69. Kadochnikova, E.Y.; Vlasov, A.A.; Alekseeva, L.I.; Didikina, I.G.; Ershova, O.B.; Zaitseva, E.M.; Korotkova, T.A.; Popova, T.A.; Sukhareva, M.L.; Taskina, E.A.; et al. The effectiveness of dynamic electroneurostimulation (DENS) in the pain management in knee osteoarthritis (results of a multicenter randomized study). Bull. Rehabil. Med. 2016, 73, 14-22. (In Russian)

70. Tkachenko, P.V.; Daminov, V.D.; Karpov, O.E. Synchronized application of the exoskeleton with functional electrostimulation in the spinal cord injury patients. Bull. Rehabil. Med. 2017, 85, 123-130. (In Russian) 


\section{Keywords}

Permanent denervated human muscle; cauda equina syndrome; home-based functional electrical stimulation; muscle co-activation; skin and muscle biopsy; color computed tomography; functional recovery 